creating the template. We reviewed PEPs from each Scottish Health Board using our template to establish whether the template could be used to help improve quality of PEPs in Scotland.

Outcomes From our consultation process, we found 14 broad themes which we felt were a priority to consider within a PEP: Initial contact; Place of Safety; Alcohol and Substance Misuse; Transport; Resolving Disputes; Assessment; Sharing Information; Missing Patients; Young People; Carers and Patients with Caring Responsibilities; Homelessness; Learning Disability and Autism; Aftercare; Use and Relevance of PEP. In total our template consisted of 63 requirements. We found that there was no heading which had not been addressed by any health board but that many requirements had not been met by all health boards.

Conclusions We have designed a template which addresses broad themes considered in most health boards already. However, not all our requirements were met by every health board. Our template could be used to raised the standard of Psychiatric Emergency Plans and therefore raise the standard of care and patient experience in Scottish Health Boards.

\section{Exception reporting}

\section{A QUALITATIVE STUDY ON EXCEPTION REPORTING IN YORKSHIRE}

Adam Dalby, Oanh Kieu Vo, Nikita Wild, Katharine Brett, Androniks Mumdzjans. Hull University Teaching Hospitals NHS Trust, Hull York Medical School

\subsection{6/leader-2020-FMLM.79}

This piece of qualitative research looks into the attitudes of managers, consultants and junior doctors towards the exception reporting process that was introduced as part of the new junior doctors' contract in December 2016.

The qualitative interviews, conducted in both structured and semi-structured format, provide a range of themes that lead to numerous recommendations for consideration by central government and lobbyists such as the British Medical Association (BMA) as to how the process could be improved.

The major themes that were identified by the interviews are; apathy, cultural issues, safety, stigma, junior-led process, training and practical issues. These are explored in some detail in the paper, with direct quotes evidencing each provided, together with a commentary.

The main recommendations include; an England-wide campaign on exception reporting to address cultural issues and stigma, more active involvement of consultants in the process, treating exception reports as a 'near miss' event from a safety perspective, lengthening timelines and introducing penalties for breaching such timelines, allowing for doctors to claim for training opportunities and address practical issues around reporting such as provision of a username and password that is the same as other IT systems in the Trusts.

Further areas of research recommended is an England-wide survey based on the themes that were elicited by the interviews, as well as the facility for further free-text comments from individuals in a survey format in order to collect quantitative data and confirm the themes with further qualitative input.

\section{Education of antimicrobial stewardship: teaching methods and their effectiveness}

\section{EDUCATION OF ANTIMICROBIAL STEWARDSHIP: TEACHING METHODS AND THEIR EFFECTIVENESS}

1,2Saarah Rana, ${ }^{1,3}$ Florence Saddler, ${ }^{1,3}$ Sally Grice. 'Queen Alexandara Hospital in Portsmouth, UK; ${ }^{2}$ Barts and the London School of Medicine, London; ${ }^{3}$ Norwich Medical School at the University of East Anglia

10.1136/leader-2020-FMLM.80

Each year thousands of lives are lost due to antibiotic-resistant infections. In order to combat the alarming rise in antibiotic resistance in the UK, guidance has been published by organisations such as the World Health Organisation and the National Institute for Clinical Excellence to help govern the use of antibiotics. In 2018 our team conducted an audit investigating antimicrobial stewardship on the general surgical wards at the Queen Alexandra Hospital, Portsmouth. This demonstrated poor compliance to the national guidance in the UK on safe antimicrobial prescribing. Our initial intervention was creating posters on the correct procedure of prescribing antibiotics in key clinical areas to promote good practice. A re-audit showed a minor improvement in compliance however this was not significant and consequently we looked into different ways of changing clinical practice. We chose to explore whether educating the prescribers about the importance of antibiotic stewardship and the clinical significance of that would be a more effective method of changing practice.

Working with the microbiology department, we formulated a teaching programme spanning four weeks. After the sessions were completed, we re-audited the surgical wards. This showed a clear improvement in compliance. This suggests the intervention was effective at impacting local clinical practice. We concluded therefore that it is possible and effective to provide relatively short and basic teaching schemes to effectively promote antimicrobial stewardship and change practice.

\section{Leadership development}

\section{AN EVALUATION OF THE CLINICAL LEADERSHIP MENTORS PROGRAMME IN THE SOUTH WEST}

${ }^{1} \mathrm{~L}$ Somerset, ${ }^{2}$ Snelling, ${ }^{2} \mathrm{H}$ Brown, ${ }^{3} \mathrm{~J}$ Thurlow, ${ }^{4} \mathrm{~L}$ Hardy, ${ }^{1} \mathrm{~S}$ Cockburn. ${ }^{1}$ Severn Deanery, SW England; ${ }^{2}$ Health Services Management Centre University of Birmingham, UK ${ }^{3}$ Associate Dean Health Education England (HEE) South West; ${ }^{4}$ NHS Leadership Academy SW England

\subsection{6/leader-2020-FMLM.81}

Aims There is an increasing evidence-base which suggests that the involvement of junior doctors in NHS leadership activities will improve services and enhance patient safety. In 2018, the HEE-South West Deanery established a Clinical Leadership 